NASA-CR-205305

\title{
Micron-sized particles detected in the vicinity of Jupiter by the Voyager plasma wave instruments
}

\author{
D. Tsintikidis \\ Hydrologic Research Center, San Diego
}

\author{
D. A. Gurnett, W. S. Kurth, and L. J. Granroth \\ Department of Physics and Astronomy, The University of lowa
}

\begin{abstract}
Wideband waveform data obtained by the plasma wave instruments onboard the Voyager 1 and 2 spacecraft have been used to study micron-sized dust particles in the vicinity of Jupiter. The technique used was developed during the flybys of Saturn, Uranus, and Neptune, and makes use of the fact that a particle striking the spacecraft at $10-20 \mathrm{~km} / \mathrm{s}$ causes a voltage pulse in the plasma wave receiver. The waveform of the voltage pulse is much different than the waveform of plasma waves and provides a highly reliable method of detecting micron-sized dust particles. Although the dust impact rate observed in the vicinity of Jupiter is much lower than the rates at Saturn, Uranus, and Neptune, the particles are easily detectable. Approximately 1200 48-second frames of wideband waveform data were examined in the vicinity of Jupiter. Dust impact signatures were found in approximately $20 \%$ of these frames. The peak impact rates are about 1 impact per second, and the peak number densities are about $10^{-5} \mathrm{~m}^{-3}$. Most of the impacts occurred near the equatorial plane at radial distances less than about $35 R_{J}$ from Jupiter. Analysis of the detection threshold indicates that the particles have masses greater than $10^{-11} \mathrm{~g}$, which corresponds to particles with diameters of a few micrometers or larger.
\end{abstract}

\section{Introduction}

The Voyager spacecraft included an instrument called the plasma wave subsystem (PWS). Although the plasma wave instrument was originally designed to study plasma waves, it has also proven useful for studying the dust environment near the outer planets. During the Voyager 2 flyby of Saturn, Scarf et al. [1982] and Warwick et al. [1982] discovered that the plasma wave and radio astronomy instruments could detect micron-sized particles striking the spacecraft. These dust impacts were subsequently studied in greater detail by Gurnett et al. [1983], who developed detailed models for the detection mechanism, and Aubier et al. [1983]. Tsintikidis et al. [1994] later improved the model of Gurnett et al. by recalculating the charge collection coefficient, which was highly uncertain in the original study.

The plasma wave instrument detects dust impacts by means of an impact ionization effect. When a small particle hits the spacecraft body at a sufficiently high velocity (i.e., 10 to 20 $\mathrm{km} / \mathrm{s}$ ), the particle and part of the surface material are vaporized and heated to a high temperatures, $10^{5 \circ} \mathrm{K}$. At this high temperature, the gas is strongly ionized, thereby producing a small cloud

Copyright 1996 by the American Geophysical Union.

Paper number 96GL00961

0094-8534/96/96GL-00961\$05.00 of plasma that expands away from the impact site. Some of the charge in the plasma cloud is collected by the plasma wave electric antenna, thereby producing a characteristic voltage waveform that can be identified in the wideband waveform data [Gurnett et al., 1983]. Since the rise time of the voltage pulse is very fast (on the order of few microseconds), it is believed that the charge collected on the antenna is due to electrons. The travel time of the ions from the spacecraft body to the antennas is on the order of milliseconds, much too long to account for the observed rise times. For more details on the coupling mechanism and the parameters involved, see Gurnett et al. [1987] and Tsintikidis et al. [1994].

Most of the early studies of the Jovian dust environment relied on the analysis of data provided by dust detectors [Van Allen, 1976; Humes, 1976; Grün et al., 1992], or by ground-based optical instruments [Jewitt and Danielson, 1981]. Images taken during the Voyager encounters added greatly to our understanding of the Jovian environment. Images obtained by the Voyager 1 wide-angle camera revealed a ring system composed of a bright narrow ring, extending from $1.72 \mathrm{R}_{\mathrm{J}}$ to $1.81 \mathrm{R}_{\mathrm{J}}\left(1 \mathrm{R}_{\mathrm{J}}=71,400\right.$ $\mathrm{km}$ ), and an interior, vertically-extended halo [Burns et al., 1984]. A Voyager 2 image led to the discovery of the gossamer ring. The gossamer ring is fainter than its counterparts and extends to about 3.5 $\mathrm{R}_{\mathrm{J}}$ [Showalter et al., 1985]. Interest in the Jovian dust environment was recently revived with the Ulysses flyby of Jupiter in February 1992 [Grün et al., 1992; Baguhl et al., 1993; Hamilton and Burns, 1993; Horanyi et al., 1993] and by the collision of comet Shoemaker-Levy (SL-9) with Jupiter in July 1994 [Grün et al,, 1994; Horanyi, 1994].

The purpose of this paper is to report in situ observations of dust in the vicinity of Jupiter using the Voyager 1 and 2 plasma wave instruments. The objectives are (1) to describe the data and the analysis techniques, (2) to derive crucial parameters such as impact rates, number densities, particle masses and sizes, and (3) to briefly discuss the results.

\section{Observations and Data Analysis}

The plasma wave instrument utilizes two antenna elements to detect the electric field of plasma waves. The antennas are used as a dipole, meaning that the instrument responds to the potential difference between the elements. Signals from the antennas are processed in two ways. First, a 16-channel spectrum analyzer is used to provide absolute spectral densities in 16 frequency channels from $10 \mathrm{~Hz}$ to $56.2 \mathrm{kHz}$. Second, a wideband waveform receiver is used to provide waveforms during selected periods over a frequency from $50 \mathrm{~Hz}$ to $12 \mathrm{kHz}$. The wideband receiver uses an automatic gain control (AGC) with a time constant of $0.5 \mathrm{~s}$. The waveforms are obtained in 48-s intervals 
Table 1. Trajectory Elements of the Voyager Encounters With Jupiter

\begin{tabular}{ccccc}
\hline Spacecraft & $\begin{array}{c}\text { Closest Approach } \\
\text { (Radial Distance and Latitude) }\end{array}$ & $\begin{array}{c}\text { Inbound Equator } \\
\text { Crossing } \\
\text { (Radial Distance) }\end{array}$ & $\begin{array}{c}\text { Outbound Equator } \\
\text { Crossing } \\
\text { (Radial Distance) }\end{array}$ & $\begin{array}{c}\text { Southernmost Point } \\
\text { (Radial Distance and Latitude) }\end{array}$ \\
\hline Voyager 1 & $\begin{array}{c}4.89 \mathrm{R}_{\mathrm{J}} \\
-5.74^{\circ}\end{array}$ & $16.96 \mathrm{R}_{\mathrm{J}}$ & $8.42 \mathrm{R}_{\mathrm{J}}$ & $4.98 \mathrm{R}_{\mathrm{J}}$ \\
Voyager 2 & $10.11 \mathrm{R}_{\mathrm{J}}$ & $19.76 \mathrm{R}_{\mathrm{J}}$ & $28.95 \mathrm{R}_{\mathrm{J}}$ & $-5.88^{\circ}$ \\
& $-9.01^{\circ}$ & & $10.17 \mathrm{R}_{\mathrm{J}}$ & $-9.10^{\circ}$ \\
\hline
\end{tabular}

or frames at a sample rate of 28,800 samples per second. The wideband receiver waveforms are useful for detecting dust impacts because the individual dust impacts can be clearly seen in the antenna voltage waveforms. For more details on the PWS instrument, see Scarf and Gurnett [1977].

Voyagers 1 and 2 both crossed the Jovian equatorial plane twice: once on the inbound pass and once on the outbound pass. For Voyager 1 the closest approach occurred on March 5, 1979, at a spacecraft event time (SCET) of 1204 SCET and a radial distance of $4.89 \mathrm{R}_{\mathrm{J}}$. For Voyager 2 the closest approach occurred on July 9, 1979, at 2228 SCET and at a radial distance of 10.11 $R_{\mathrm{J}}$. Table 1 provides a summary of the basic trajectory parameters for both encounters. Because of the very high levels of plasma wave noise, no attempt was initially made to study dust impacts, even though it was realized that some impacts were probably present.

A typical dust impact in the PWS wideband waveform data consists of an abrupt voltage pulse with a rise time of a few microseconds, followed by a complex, oscillatory recovery lasting up to several milliseconds (see Figure 4 of Tsintikidis et al. [1994]). In all the aforementioned studies, a computer algorithm was used to detect the pulses. The algorithm calculated the slopes between two contiguous pairs of points and required the slopes to be above a selected threshold. The threshold was adjusted in such a way as to provide good agreement with visual identification of the impact events. In order to avoid false events generated by the complex recovery waveform, a dead time was introduced after each event, that depended on the magnitude of the initial slope. The detection scheme worked well when the level of plasma wave activity was low, as it was during the ring plane crossings of Saturn, Uranus, and Neptune. However, in the presence of strong plasma wave activity, such as occurred at Jupiter, the algorithm tended to produce many false alarms.

Jupiter possesses the largest and most complex magnetosphere in the solar system and has a complex and variable plasma wave spectrum. During the Voyager Jupiter flybys the plasma wave activity was very rarely low even at large distances. Consequently most of the dust impacts were embedded in a noisy background of plasma wave activity. Figure 1 shows a comparison of two impacts: the top impact emerges from a constant lowlevel background while the bottom impact emerges from a complex background of plasma wave activity. Most of the impacts detected in the course of this study were similar to the second impact. Many false alarms occurred when plasma wave activity caused contiguous pairs of points to exceed the preset slope threshold. To minimize such false alarms the detection criterion was altered by using three pairs of contiguous points with the slope threshold set higher than previous studies. The revised detection criterion decreased the false alarm rate significantly, but not enough to allow completely automatic processing. After considerable experimentation it was decided to visually inspect all the events detected by the algorithm, and thereby decide by eye which events are real. Visual inspection slowed the overall detection process considerably, but provided a high level of confidence that the signals identified were due to actual dust impacts.

Approximately 120048 -second frames of wideband data were examined from both encounters. These frames constituted all of the available data. During the equatorial plane crossings the relative velocity between the spacecraft and particles in Keplerian orbits was $16.1 \mathrm{~km} / \mathrm{s}$ for Voyager 1 and $11.6 \mathrm{~km} / \mathrm{s}$ for Voyager 2. Most of the data examined were within a few degrees of the equatorial plane at distances ranging from about 5 to $28 \mathrm{R}_{\mathrm{J}}$ for Voyager 1 and from about 10 to $35 R_{\mathrm{J}}$ for Voyager 2 . A few frames were obtained at the southernmost parts of the trajectories (about $-6^{\circ}$ and $-8^{\circ}$ latitude for Voyager 1 and 2, respectively).

In the previous dust impact studies, usually no more than a few consecutive events (e.g., about 5 to 10) had the same polarity. During the Jupiter study several cases were discovered in which a large number of consecutive events of the same polarity were detected, i.e., about 50 pulses all negative or all positive. These observations contrasted with the fact that at the other planets the impacts were always of random polarity (that is, the signs of successive pulses were statistically uncorrelated). A random polarity is expected since impacts occur at various random locations around the spacecraft, relative to the PWS dipole antenna. The cases in which a large number of events of the same polarity were detected are believed to be spacecraft electrostatic discharges (accumulation of charge at some locations on the spacecraft body or instruments that leads to a discharge) and were discarded. It is likely that discharge conditions occur at one or more specific locations on the spacecraft and that the fixed position relative to the PWS antennas would yield pulses of the same polarity for a given discharge site. For the remaining impacts the ratio of the number of negative impulses to the number of positive impulses was 1.06 , which is comparable to the ratio of 1.13 for Saturn and 0.88 for Uranus [Gurnett et al., $1983 ; 19871$.

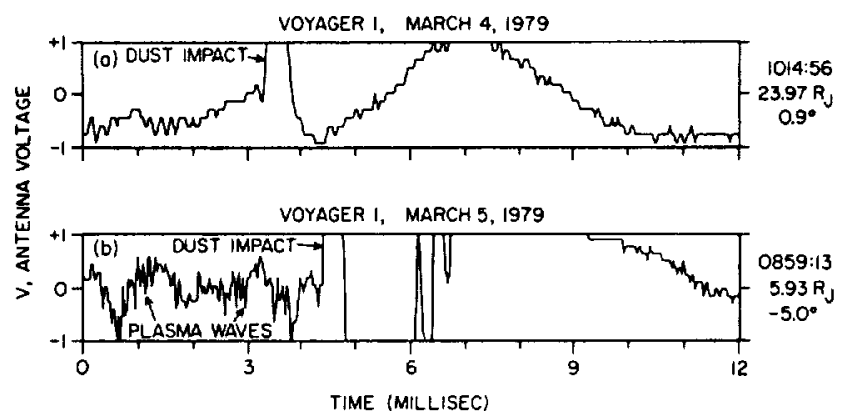

Figure 1. Top panel: an impact occurring when the background is "quiet." Bottom panel: an impact occurring in the presence of noisy background plasma wave activity. Most of the observed impacts are similar to the one in the bottom panel, which makes their detection more challenging. 


\section{Results and Discussion}

The majority of the data frames examined $(\sim 900)$ were from the Voyager 1 encounter with Jupiter. The maximum impact rate in the Voyager 1 data was about $0.9 \mathrm{~s}^{-1}$ and occurred when the spacecraft was at a radial distance of about $23.5 \mathrm{R}_{\mathrm{J}}$ and a latitude of $1^{\circ}$ north. In general most of the impacts occurred when the spacecraft was within a few degrees of the Jovian equatorial plane. A small number of impacts were observed during the southernmost part of its trajectory (near closest approach). The impact rate profile during the Voyager 1 flyby of Jupiter is shown in the top panel of Figure 2. The vertical bars above the top panel indicate the data frames analyzed. The impact rate was calculated for each 48-second frame for which one or more impacts were observed. As can be seen, the number of vertical bars is larger than the number of impact rate points: about $20 \%$ of the 48-second frames contained impacts.

The maximum impact rate for the Voyager 2 encounter was somewhat less than the impact rate for the Voyager 1 encounter, about $0.4 \mathrm{~s}^{-1}$. The maximum impact rate occurred when the spacecraft was at a radial distance of about $22 \mathrm{R}_{\mathrm{J}}$ and at a latitude of $1^{\circ}$ north. Again, the majority of the impacts were detected within one degree of the equatorial plane. The impact rate reached a maximum value just before the spacecraft reached the southernmost point of its trajectory. The Voyager 2 impact rate profile is shown in the top panel of Figure 3.

Once the impact rate, $\mathbf{R}$, is known, the particle number density, $n$, can be derived from the equation

$$
\mathrm{R}=\mathrm{nUA}_{\mathrm{S} / \mathrm{C}}
$$

where $A_{S / C}$ is the spacecraft effective cross-sectional area, and
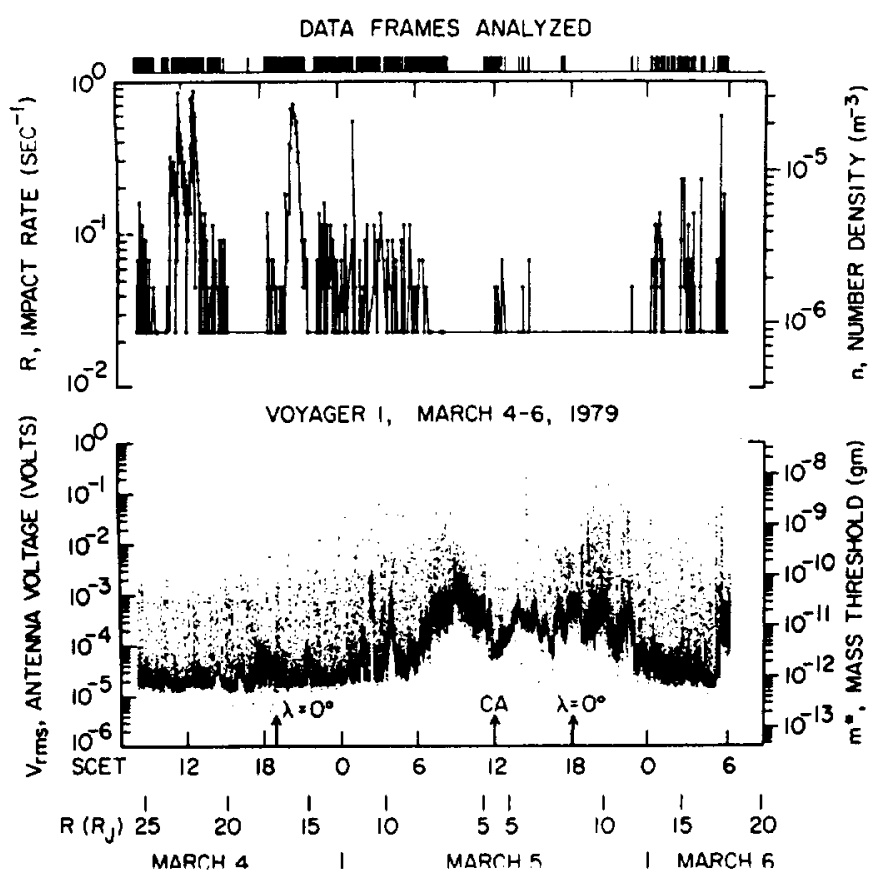

Figure 2. A comparison of the impact rate, $R$, and the rms antenna voltage, $V_{\text {rms }}$, profiles during the Voyager 1 encounter with Jupiter. Each vertical bar at the top represents one highresolution data frame. The scale on the right of the bottom panel shows the mass threshold, $\mathrm{m}^{*}$, for detecting impacts. The rms voltage values are irrelevant when there are no impacts. The arrows point to the inbound equatorial crossing $\left(\lambda=0^{\circ}\right)$, closest approach (CA), and outbound equatorial crossing $\left(\lambda=0^{\circ}\right)$, respectively.
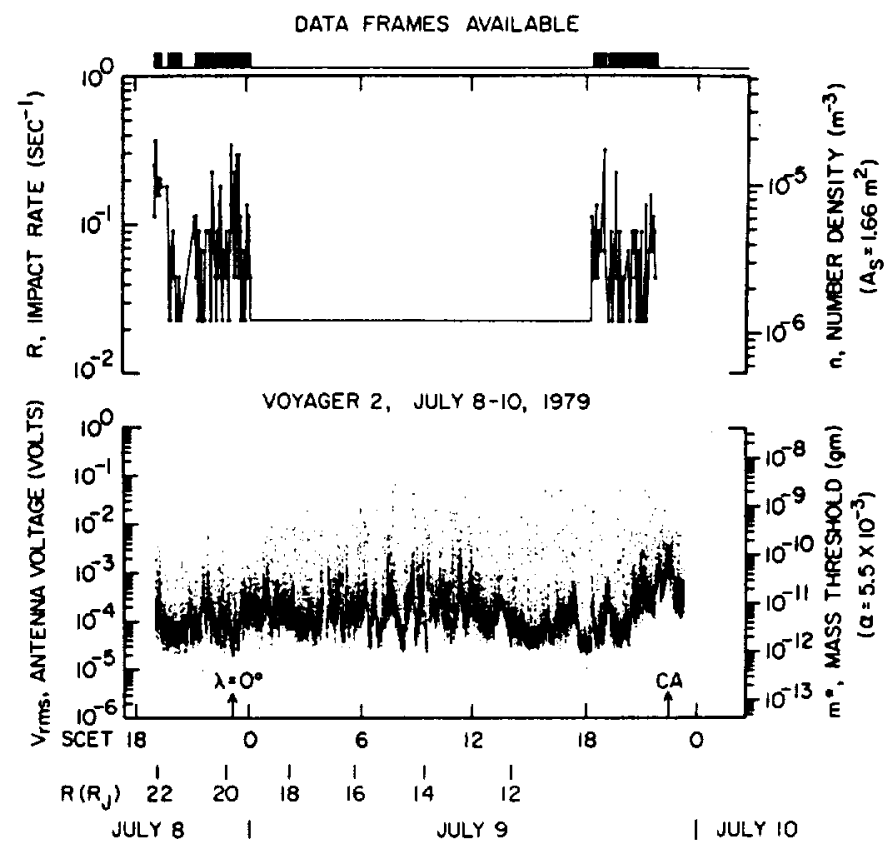

Figure 3. Same as in Figure 2, but for the Voyager 2 encounter with Jupiter.

$\mathrm{U}$ is the relative speed between the particles and the spacecraft. The effective area has been estimated by Gurnett et al. [1983] to be $1.66 \mathrm{~m}^{2}$. The relative speed did not change significantly from its value during the equatorial crossings as long as the spacecraft were at great distances and within a few degrees of the equatorial plane, which was indeed the case for most of the data analyzed. The number density profiles are shown in the top panels of Figures 2 and 3 . The maximum particle densities were calculated to be $\mathrm{n}_{\max }(\mathrm{V} 1)=3.4 \times 10^{-5} \mathrm{~m}^{-3}$ and $\mathrm{n}_{\max }(\mathrm{V} 2)=2.1 \times 10^{-5} \mathrm{~m}^{-3}$, for Voyagers 1 and 2, respectively.

The bottom panels of Figures 2 and 3 show the rms antenna voltage profiles obtained from the 16-channel spectrum analyzer during the Voyager 1 and 2 flybys. The threshold mass for indetecting an impact can be calculated from the rms antenna voltage. The threshold mass is the minimum particle mass required to produce a voltage pulse above the background noise level and is given by

$$
\mathrm{m}^{*}=\left(\frac{\beta \mathrm{C}_{\mathrm{A}}}{\alpha \mathrm{k}}\right) \mathrm{V}_{\mathrm{rms}}
$$

은

The above equation was first derived by Gurnett et al. [1983] and gives the lower mass limit for a detected dust impact. The nominal parameters are: antenna capacitance, $C_{A}=90 \mathrm{pF}$, collection coefficient, $\alpha=0.0055$, yield constant, $\mathrm{k}=0.21 \mathrm{C} / \mathrm{g}$, and $\beta=0.51$. The above values were used by Tsintikidis et al. [1995] and are average values for all of the encounters. For a typical $\mathrm{V}_{\mathrm{rms}}$ value of $3.0 \times 10^{-4} \mathrm{~V}$, the threshold mass, $\mathrm{m}^{*}$, is approximately $1.2 \times 10^{-11} \mathrm{~g}$. The corresponding particle size is $1.4 \mu \mathrm{m}$ for an ice particle or $1.1 \mu \mathrm{m}$ for silicates (density of 2 $\mathrm{g} / \mathrm{cm}^{3}$ ). Most likely, smaller particles exist in the region sampled. However, it is highly unlikely that the instruments are sensitive enough to respond to submicron dust impacts. Figures 2 and 3, suggest that the largest impact rates occur in regions of low plasma wave activity, indicating that the impact signals, especially the ones resulting from small particles, are probably being masked in regions of high plasma wave activity. When the plasma wave activity is high, the AGC reduces the gain of the 


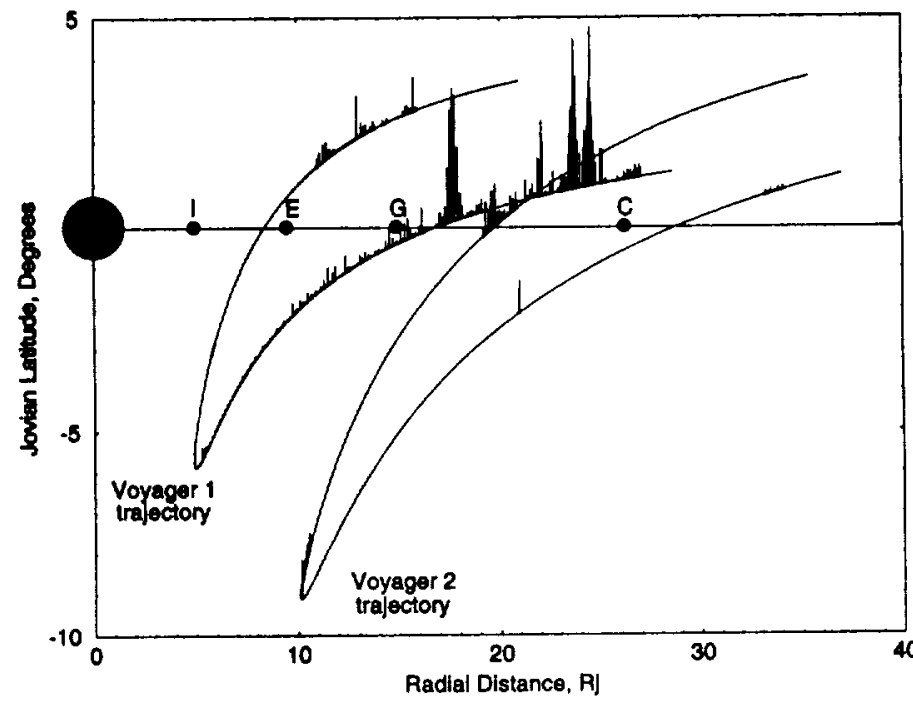

Figure 4. A comparison of impact rates along the Voyager $I$ and 2 trajectories by Jupiter. The vertical lines indicate the impact rates. The tallest vertical line corresponds to an impact rate of $0.9 \mathrm{~s}^{-1}$. The initials I, E, G, C stand for the four Galilean moons (Io, Europa, Ganymede, and Callisto).

wideband receiver, thereby making the dust impacts harder to detect.

Since the data were obtained from only two flybys, the Voyager observations provide only a limited survey of the Jovian dust environment. The results of both encounters can be summarized in Figure 4 . The highest impact rates occurred when the spacecraft were close to the equatorial plane and between Ganymede and Callisto. The peak in the impact rates near these large moons suggests that the moons may play a role in the generation of dust, similar to the role that Dione plays at Saturn (Tsintikidis et al. [1995] and references therein). In general, the Jovian impact rates, number densities, and masses are much smaller than those detected at the other planetary encounters, but the equatorial crossing distances are much greater. For example, at Neptune $\mathrm{R}_{\max }$ was $421 \mathrm{~s}^{-1}, \mathrm{n}_{\max }$ was $1.1 \times 10^{-2} \mathrm{~m}^{-3}$, and $\mathrm{m}^{*}$ ranged upwards from $10^{-10} \mathrm{gm}$ [Gurnett et al., 1991]. On the other hand, the masses of the particles detected are much larger than the particles detected by Ulysses, which fall in the range from approximately $10^{-15}$ to $10^{-14} \mathrm{gm}$ [Baguhl et al., 1993], although their distances were a few hundred to a few thousand Jovian radii.

The Voyager observations are particularly important because they were obtained in 1979, well before the breakup and collision of Shoemaker/Levy-9. Given recent reports of Jovian dust storms encountered by Galileo, such baseline measurements may help determine whether the dust detected by Galileo was introduced by the SL-9 collision.

Future work involves determination of the particle sources, the particle size distribution (if possible), and the possible connection with nearby moons and/or Jupiter's faint rings. Galileo arrived at and was inserted into orbit around Jupiter on December 7, 1995. Because Galileo will orbit Jupiter many times, the opportunity exists to sample the Jovian environment with a better spatial and temporal resolution than is available from the Voyager encounters. Consequently, a more complete picture of the Jovian dust environment will emerge.
Acknowledgments. The authors wish to thank Jay Ansher for useful discussions. Work was supported by contracts 959193 and 958779 with the Jet Propulsion Laboratory.

\section{References}

Aubier, M. G., N. Meyer-Vernet, and B. M. Pedersen, Shot noise from grain and particle impacts in Saturn's ring plane, Geophys. Res. Letr., $10,5,1983$

Baguhl, M., E. Grün, G. Linkert, D. Linkert, and N. Siddique, Identification of "small" dust impacts in the Ulysses dust detector data, Planet. Space Sci. 41, 1085, 1993.

Burns, J. A., M. R. Showalter, and G. E. Morfill, The ethereal rings of Jupiter and Saturn, in Planetary Rings, p. 200, edited by R. Greenberg and A. Brahic, University of Arizona Press, Tucson, 1984.

Gurnett, D. A., E. Grün, D. Gallagher, W. S. Kurth, and F. L. Scarf, Micron-sized particles detected near Saturn by the Voyager plasma wave instrument, Icarus, 53, 236, 1983

Gurnett, D. A., W. S. Kurth, F. L. Scarf, J. A. Burns, J. N. Cuzzi, and E. Grün, Micron-sized particles detected near Uranus by the Voyager plasma wave instrument, J. Geophys. Res., 92, 14,959, 1987.

Gurnett, D. A., W. S. Kurth, L. J. Granroth, S. C. Allendorf, and R. L. Poynter, Micron-sized particles detected near Neptune by the Voyager plasma wave instrument, J. Geophys. Res., 96, 19,177, 1991.

Grün, E., H. A. Zook, M. Baguhl, H. Fechtig, M. S. Hanner, J. Kissel, B. A. Lindblad, D. Linkert, G. Linkert, I. B. Mann, J. A. M. McDonnell, G. E. Morfill, C. Polanskey, R. Riemann, G. Schwehm, and N. Siddique, Ulysses dust measurements near Jupiter, Science, $257,1550,1992$

Grün, E., D. P. Hamilton, M. Baguhl, R. Riemann, M. Horanyi, and C. Polanskey, Dust streams from comet Shoemaker-Levy 9?, Geophys. Res. Lett., 2I, 1035, 1994.

Hamilton, D. P., and J. A. Burns, Ejection of dust from Jupiter's gossamer ring, Nature, 364, 695, 1993.

Horanyi, M., G. Morfill, and E. Grün, The dusty ballerina skirt of Jupiter, J. Geophys. Res., 98, 21,245, 1993.

Horanyi, M., New Jovian ring?, Geophys. Res. Lett., 21, 1039, 1994.

Humes, D. H., The Jovian meteoroid environment, in Jupiter, p. 1052, edited by T. Gehrels, University of Arizona Press, Tucson, 1976.

Jewitt, D. C., and G. E. Danielson, The Jovian ring, J. Geophys. Res., $86,8691,1981$.

Scarf, F. L., and D. A. Gurnett, A plasma wave investigation for the Voyager mission, Space Sci. Rev., 21, 289, 1977.

Scarf, F. L., D. A. Gurnett, W. S. Kurth, and R. L. Poynter, Voyager 2 plasma wave observations at Saturn, Science, 215, 287, 1982.

Showalter, M. R., J. A. Burns, J. N. Cuzzi, and J. B. Pollack, Discovery of Jupiter's 'gossamer' ring, Nature, 316, 526, 1985.

Tsintikidis, D., D. A. Gurnett, L. J. Granroth, S. C. Allendorf, and W. $S$. Kurth, A revised analysis of micron-sized particles detected near Saturn by the Voyager 2 plasma wave instrument, J. Geophys. Res., $99,2261,1994$.

Tsintikidis, D., W. S. Kurth, D. A. Gurnett, and D. D. Barbosa, A study of dust in the vicinity of Dione using the Voyager 1 plasma wave instrument, J. Geophys. Res., 100, 1811, 1995.

Van Allen, J. A., High-energy particles in the Jovian magnetosphere, in Jupiter, p. 928, edited by T. Gehrels, University of Arizona Press, Tucson, 1976.

Warwick, J. W., D. R. Evans, J. H. Romig, J. K. Alexander, M. D. Desch, M. L. Kaiser, M. Aubier, Y. Leblanc, A. Lecacheux, and B. M. Pedersen, Planetary radio astronomy observations from Voyager 2 near Saturn, Science, 215, 582, 1982.

L. J. Granroth, D. A. Gurnett, W. S. Kurth, Department of Physics and Astronomy, The University of Lowa, Iowa City, IA 52242. (e-mail: larry-granroth@uiowa.edu; donald-gurnett@uiowa.edu; william-kurth@ uiowa.edu)

D. Tsintikidis, Hydrologic Research Center, 12780 High Bluff Drive, Suite 260, San Diego, CA 92130 (e-mail: ditsinti@hml06.ucsd.edu).

(Received January 8, 1996; accepted March 4, 1996.) 